\title{
Zur Kenntnis von Elektrodenvorgängen. Ueber den Einfluss von Temperaturerhöhung und Depolarisatoren auf die Abscheidungsform des Nickels
}

\author{
von
}

Hans Stăger.

(6. VII. 20.)

\section{Einleitung.}

Bekanntlich wechselt die Form eines elektrolytischen Niederschlages unter vergleichbaren Bedingungen stark sowohl mit der Art des Metalls als auch beim gleichen Metall mit der Natur und Beschaffenheit des Elektrolyten. Letzteres gilt nicht nur, wenn die Badflüssigkeit mit Zusätzen versehen wird, von denen man sich eine Beeinflussung der Faktoren im Krystallisationsprozess vorstellen kann, oder wenn an Stelle einfacher Metallsalze Komplexverbindungen gewählt werden, sondern tritt oft schon bei gewöhnlichen Salzen durch blosse Änderung des Anions in die Erscheinung, also auch dann, wenn der Vorgang der Metallabscheidung in der Entladung eines einfachen Metallions besteht und Nebenwirkungen durch stofffremde Zersetzungsprodukte des Elektrolyten nicht wahrscheinlich gemacht werden können.

In derartigen Fällen wird man anderen, einer bestimmten Abscheidungsform parallel gehenden Erscheinungen erhöhte Aufmerksamkeit zu schenken haben, und zu solchen gehört die Erfahrung, dass bei manchen Metallen eine ausgesprochene Hemmung der kathodischen Abscheidung, d. h. ein anormal hohes Entladungspotential, mit der sonst ungewöhnlichen Ausbildung glatter und höchstens feinkörniger Niederschläge zusammenfällt, wie dies besonders bei den Metallen der Eisengruppe beobachtet wird, während Änderung der Bedingungen die beiden auffälligen Besonderheiten mitunter gleichzeitig zum Verschwinden bringt.

Diesem Fingerzeige folgend und in der Vermutung, dass hier eine ursächliche Beziehung zwischen Niederschlagsform und kathodischer Polarisation bestehe, haben Kohlschütter und Vuilleumier ${ }^{1}$ )

1) Z. El. Ch. 24, 300 (1918). 
die Abscheidung von Nickel in ihren Anfangsstadien zu beobachten versucht, indem sie von der weiteren Eigentümlichkeit des Elektrolytnickels Gebrauch machten, dass in ihm bei seiner Bildung Spannungen auftreten, die beim Anwachsen eines Niederschlags das technisch so lästige Abblättern und Springen der kathodischen Überzüge verursachen. Bei sehr dünnen Schichten konnte dieser mechanische Effekt auf eine biegsame Unterlage übertragen und durch geeignete Anbringung eines Zeigers auf einer Skala sichtbar gemacht werden; so liess sich nachweisen, dass im Nickelniederschlag von seinem ersten Auftreten an eine Kontraktion vor sich geht, die nach Betrag und Verlauf von verschiedenen Umständen beeinflusst wird, aber gut reproduzierbar und ziemlich empfindlich mit dem so geschaffenen „Kontraktometer" verfolgbar ist.

Die Analyse der Erscheinung führte zu der Auffassung, dass das Metall zuerst in Gestalt einer hochdispersen, zusammenhängenden Schicht erscheint, die sich stetig durch einen Sinterungs- bezw. Koagulationsvorgang unter Teilchenvergrösserung kontrahiert und ihre Unterlage, auf der sie fest aufliegt, mitzieht. Einem hochdispersen Material muss nun ein höherer elektrolytischer Lösungsdruck zukommen als dem kompakten Metall, sodass auch eine höhere Abscheidungsspannung erforderlich ist, solange der Niederschlag in dieser Form auftritt. So angesehen haben also die beiden auffälligen Tatsachen die gleiche Ursache, und es hat sich gezeigt, dass alle Beobachtungen über das Potential und die mechanische Veränderung von Elektrolytnickel unter diesem Gesichtspunkt eine Erklärung finden. Für die Ausbildung der besonderen Form aber wurde die Wasserstoffhaut verantwortlich zu machen versucht, die an einer nickelabscheidenden Elektrode notwendig vorauszusetzen ist. In dieser komprimierten Gasschicht schlägt sich das Metall wie in einem stark viskosen Dispersionsmittel nieder, und in ihr vollzieht sich auch die Dispersitätsverminderung, die in der Kontraktion der Metallhaut zum Vorschein kommt. Der Gashaut aber einen solchen bestimmenden Einfluss für die Abscheidungsform zuzuweisen, wurde besonders durch eigentiumliche Verzögerungserscheinungen nahegelegt, die kaum anders deutbar sind als dadurch, dass sie mit einem plötzlichen Zerreissen der über ein gewisses Mass verdickten Gasschicht in Verbindung gebracht werden. 
Wenn sich nun auch durch die hier ganz kurz wiedergegebene Auffassung ein einleuchtender Zusammenhang zwisehen Polarisation und Abscheidungsform ergibt, aus dem zahlreiche Einzelheiten in den Erscheinungen zwanglos folgen, so war es doch erwïnscht, ihre Grundlage einer weiteren experimentellen Prifung $\mathrm{zu}$ unterziehen.

Eine solche erschien zunächst auf doppelte Weise möglich.

Einmal nämlich war die Bildung bezw. die Veränderung von Nickelniederschlägen bei Bedingungen zu verfolgen, unter denen eine Herabsetzung der Polarisation erzielt wird, denn im Sinne der obigen Anschauung musste dies von einer Abnahme der spontanen Veränderungsfähigkeit des Niederschlags, wie sie am Kontraktometer abgelesen wird, begleitet sein. Hierfür kam in erster Linie eine Erhöhung der Temperatur, bei der die Elektrolyse durchgeführt wird, in Frage, denn nach Versuchen von Schweitzer ${ }^{1}$ ) geht die Überspannung der Nickelabscheidung mit steigender Temperatur herab. Andrerseits nimmt erfahrungsgemäss die Krystallisationsfähigkeit der Nickelniederschläge durch Steigerung der Temperatur stark zu, während bei den anderen elektrolytisch abgeschiedenen Metallen der Eisengruppe eine merkliche Verminderung des Aufnahmevermögens für Wasserstoff unter denselben Bedingungen festgestellt wurde, die daher auch beim Nickel wahrscheinlich ist, und die Neigung zum Blättern sich gleichzeitig stark vermindert ${ }^{2}$ ).

Dies würde im Sinne der skizzierten Anschauung so zu deuten sein, dass die Wasserstoffschicht an der Elektrode bei höherer Temperatur entweder nicht mehr in genügender Dicke entsteht, oder wenigstens nicht mehr in gleichem Masse als dispersitätsvermehrend in Funktion tritt, denn alle die erwähnten anormalen Erscheinungen sind ja nach jener Auffassung durch die Ausbildung einer hochdispersen Schicht veranlasst, die ihrerseits durch jene Wasserstoffhaut verursacht ist und von dieser beim Heranwachsen natürlich leicht einen Teil in sich begraben kann. Da auch die beobachtete Kontraktion an diese Form gebunden ist, war zu vermuten, dass auch sie verringert oder aufgehoben werden würde, wenn die Bildungstemperatur erhöht

1) 7. El. Ch. 15, 602 (1909).

2) vgl. Foerster, Wlektroch. wässr. Lsgn., 2. Aufl., S. 325, Leipzig (1915). 
wird, denn ein schon feinkörniger oder gar deutlich krystalliner Niederschlag bietet nicht mehr die Voraussetzung für eine Schrumpfung durch Teilchenvereinigung oder -vergrösserung.

Kohlschütter und Vuilleumier haben über das mechanische Verhalten der Nickelniederschläge unter dem Einfluss der Temperatur schon einige vorläufige Versuche gemacht, stiessen jedoch auf Komplikationen, die von einer Veränderung in der Beschaffenheit des Elektrolyten herzukommen schienen, wenri auch das Ergebnis in der erwarteten Richtung lag; eine genauere Verfolgung der Verhältnisse bei verschiedenen Temperaturen war daher notwendig.

In zweiter Linie war zu untersuchen, wie sich die Niederschläge verhielten, wenn man die Ausbildung der Wasserstoffhaut, die als die eigentliche Ursache der hochdispersen Metallablagerungen angesehen wird, durch kathodische Depolarisatoren unterdrückt, denn es musste dann auch der am Kontraktometer messbare Effekt verringert oder aufgehoben werden.

Da nach der Untersuchung von Schweitzes die anormalen Potentiale bei der kathodischen Abscheidung mitsamt ihrer Abhängigkeit von Stromdichte und Temperatur ein auffälliges Gegenstück in Hemmungen bei der anodischen Auflösung haben, so war es schliesslich von Wichtigkeit, auch das Verhalten von Nickelanoden bei der Polarisation zu untersuchen, doch sollen die hierauf sich beziehenden Versuche erst im Zusammenhang mit anderen dazu gehörenden Beobachtungen in einer folgenden Mitteilung wiedergegeben werden, während die vorliegende die beiden erstgenannten Fragen behandelt.

\section{Untersuchungsmethode.}

a) Apparat. Für die Versuche wurde der schon von Kohlschütter und Vuilleumier gebrauchte Apparat benutzt, der in seinem wichtigsten Teile, der als Kathode dienenden Platinlamelle, unverändert gelassen, aber in Einzelheiten noch verbessert wurde. Die Lamelle hatte demgemäss eine Länge von $6,5 \mathrm{~cm}$ und eine Breite von $2 \mathrm{~cm}$; sie wurde oben auf $1 / 2 \mathrm{~cm}$ zwischen Metallbacken festgehalten und tauchte bis zur Hälfte ihrer freien Fläche, d. h. $3 \mathrm{~cm}$ tief, in die Fliussigkeit; zu ihrer Herstellung hatten Bleche von $0,1 \mathrm{~mm}$ (Elektrode I) bezw. 0,05 mm Stärke (Elek- 
trode II) gedient. Hinsichtlich der sonstigen Einrichtung und Handhabung des Apparats muss auf die zitierte Abhandlung verwiesen werden.

Als isolierender Überzug für die Rückseite der Elektrode erwies sich Vaseline von geeigneter Konsistenz auch bei höheren Temperaturen als brauchbar. Der Zeiger an der Elektrode war $30 \mathrm{~cm}$ lang; die Ablesungen an der Skala geschahen mit einer Lupe, wodurch Verschiebungen von der Grössenordnung $0,1 \mathrm{~mm}$ ziemlich sicher geschätzt werden konnten. Die Elektroden wurden vor jedem Versuch mit Alkohol von ihrem Vaselinüberzug befreit, sorgfältig in Salpetersäure ausgekocht, ausgeglüht und auf einer Glasplatte geglättet.

Der Apparat erwies sich in zahllosen Versuchen als absolut zuverlässig. Selbst bei tagelangem Verbleib in einer stromlosen Flüssigkeit änderte sich die Zeigerstellung nicht im geringsten, namentlich aber erfolgte die Verschiebung bei Stromdurchgang durchaus eindeutig nach rechts oder links, entsprechend einer Kontraktion oder Dehnung, sodass der kleinste Effekt einer mechanischen Veränderung in der wirksamen Fläche mit Sicherheit dem Sinne nach festgestellt werden konnte.

b) Anordnung. Als Stromquelle dienten zwei grössere Akkumulatoren, die durch einen als Gefälldraht geschalteten Schieberwiderstand von ca. $400 \mathrm{Ohm}$ kurzgeschlossen waren. Von ihm wurde der Strom durch ein Präzisionsmilliamperemeter und eine Wippe zur Zelle geführt; die letztere bestand aus einem rechteckigen Glastrog von $100 \mathrm{~cm}^{3}$ Inhalt. Als Anode wurde entweder ein Nickelblech oder, bei Sulfatlösungen, ein Platinblech verwendet. Ein Millivoltmeter war zur Verfolgung der Klemmenspannung ausschaltbar an den Elektroden angelegt.

c) Lösungen. Die Elektrolyte waren so gewählt, dass der Anschluss einerseits an frühere Versuche über die Polarisation und das Blättern des Metalls an Nickelelektroden von Foerster, Riedel ${ }^{1}$ ) und Schweitzer, andrerseits an die Versuche von Kohlschütter und Vuilleumier gewonnen war. Demgemäss kamen die folgenden Badfluissigkeiten zur Anwendung:

1. 0,25-n. Nickelchlorid, 0,02-n. Salzsäure

2. 0,25-n. Nickelchiorid, 0,1-n. Essigsäure

1) Z. El. Ch. 21, 5 (1915); 22, 281 (1916). 
3. n. Nickelchlorid, 0,5\% Borsäure

4. n. Nickelchlorid, n. Ammoniumchlorid

5. n. Nickelsulfat

6. 0,25-n. Nickelsulfat, 0,1-n. Essigsäure

7. n. Nickelsulfat, $0,5 \%$ Borsäure.

Die von Fall zu Fall benutzten Lösungen wurden aus Stammlösungen hergestellt, die durch Fällung mit basischem Nickelkarbonat eisenfrei gemacht waren; auch Kupferion und Zinkion waren in ihnen nicht mehr nachweisbar, dagegen enthielten sie noch geringe Mengen Kobaltion.

\section{Versuche.}

\section{A. Einfluss der Temperatur auf die Kontraktion des Nickelniederschlags.}

Die Elektrolysierzelle war bei diesen Versuchen in einem grossen Becherglase, das als Wasserthermostat diente, auf einem Glasdreifuss aufgestellt. Die Temperatur wurde sowohl im Wasserbade als auch in der Zelle beobachtet und war während der Versuchsdauer leicht auf $0,5^{0}$ konstant zu halten, da zur Feststellung des charakteristischen Verhaltens eines Niederschlags meist eine Elektrolyse von $20-30^{\prime}$ bei Stromdichten von $5-15 \mathrm{M}$. A. $/ \mathrm{cm}^{2}$ genügte.

In den folgenden Tabellen gibt die erste Spalte jeder Abteilung die Zeit nach Beginn der Elektrolyse, die zweite die Anzahl der Skalenteile, die der Zeiger zurückgelegt hat, die dritte die Differenz für je zwei Ablesungen an.

a) Versuche in $0,25-n . \mathrm{NiCl}$-Lösungen mit Salzsöureund Essigsä̈urezusatz.

Die Resultate der Beobachtungen, die bei Zimmertemperatur von ca. $18^{\circ}$, bei 50 und $70^{\circ}$ angestellt wurden, sind in den Tabellen $I$ und II und Fig. 1 und 2 vereinigt. 
Tabelle I.

0,25-n. Ni Cl $]_{2}, 0,02-n$. HCI. Kathode I. $\mathrm{D}_{\mathrm{K}}=5$ Milliamp. $/ \mathrm{cm}^{2}$

\begin{tabular}{|c|c|c|c|c|c|c|c|c|}
\hline \multicolumn{3}{|c|}{ NP. 9} & \multicolumn{3}{|c|}{ Nr. 12} & \multicolumn{3}{|c|}{ Nr. 15} \\
\hline \multicolumn{3}{|c|}{$18^{0}$} & \multicolumn{3}{|c|}{$50^{\circ}$} & \multicolumn{3}{|c|}{$70^{\circ}$} \\
\hline $0^{\prime}$ & 0,00 & $\operatorname{Diff} / 2^{\prime}$ & $0^{\prime}$ & 0,00 & Diff/ $/ 2^{\prime}$ & $0^{\prime}$ & 0,00 & $\operatorname{Dift} / 2^{\prime}$ \\
\hline $2^{\prime}$ & 0,86 & 0,86 & $2^{\prime}$ & 0,31 & 0,31 & $2^{\prime}$ & 0,28 & 0,28 \\
\hline $4^{\prime}$ & 1,45 & $0, \overline{5} 9$ & $4^{\prime}$ & $0, \overline{0} \overline{0}$ & 0,24 & $4^{\prime}$ & 0,40 & 0,12 \\
\hline $6^{\prime}$ & 1,89 & 0,44 & $6^{\prime}$ & 0,76 & 0,21 & $6^{\prime}$ & 0,50 & 0,10 \\
\hline $8^{\prime}$ & 2,24 & 0,35 & $8^{\prime}$ & 0,96 & 0,20 & $8^{\prime}$ & 0,61 & 0,11 \\
\hline $10^{\prime}$ & 2.57 & 0,33 & $10^{\circ}$ & 1,24 & 0,28 & $10^{\prime}$ & 0,72 & 0,11 \\
\hline $12^{\prime}$ & 2,88 & 0,31 & $12^{\prime}$ & $1,5 \bar{\jmath}$ & 0,33 & $12^{\prime}$ & 0,81 & 0,09 \\
\hline $14^{\prime}$ & 3,13 & 0,25 & $14^{\prime}$ & 1,99 & 0,42 & $14^{\prime}$ & 0,90 & 0,09 \\
\hline $16^{\prime}$ & 3,34 & 0,21 & $16^{\prime}$ & 2,22 & 0,23 & $16^{\prime}$ & 0,99 & $\overline{0}, 09$ \\
\hline $18^{\prime}$ & 3,52 & 0,18 & $18^{\prime}$ & 2,43 & 0,19 & $18^{\circ}$ & 1,05 & 0,06 \\
\hline $20^{\prime}$ & 3,68 & 0,16 & $20^{\prime}$ & 2,58 & 0,15 & $20^{\circ}$ & 1,13 & 0,08 \\
\hline
\end{tabular}

Tabelle II.

0,25-n. NiCi. , 0,1-n. Essigsäure. Kathude I. $\mathrm{D}_{\mathrm{K}}=\tilde{\mathrm{o}}$ Milliamp/em²

\begin{tabular}{|c|c|c|c|c|c|c|c|c|}
\hline \multicolumn{3}{|c|}{$\mathrm{NP} \cdot 1$} & \multicolumn{3}{|c|}{$\mathrm{Nl}^{\circ} 4$} & \multicolumn{3}{|c|}{ Nr. 7} \\
\hline \multicolumn{3}{|c|}{$18^{\circ}$} & \multicolumn{3}{|c|}{500} & \multicolumn{3}{|c|}{$70^{\circ}$} \\
\hline$\gamma^{\prime}$ & 0,00 & bifr/2" & $0^{\prime}$ & 0,00 & $\operatorname{Dilf} / \Omega^{\prime}$ & $0^{\prime}$ & 0.00 & $\operatorname{bifl} / 2^{\prime}$ \\
\hline $2^{\prime}$ & 0,66 & 0,66 & $2^{\prime}$ & 0,39 & 0,39 & $2^{\prime}$ & 0,20 & 0,20 \\
\hline $4^{\prime}$ & 1,11 & 0,48 & $4^{\prime}$ & 0,62 & 0,23 & $4^{\prime}$ & 0,32 & 0,12 \\
\hline $6^{\prime}$ & $1, \tilde{53}$ & 0,39 & $6^{\prime}$ & 0,81 & 0,22 & $6^{\circ}$ & 0,46 & 0,14 \\
\hline $8^{\prime}$ & 1,91 & 0,38 & $8^{\prime}$ & 1,03 & 0,19 & $8^{\prime}$ & 0,60 & 0,14 \\
\hline $10^{\prime}$ & 2,23 & 0,32 & $10^{\prime}$ & 1,18 & $0,1 \check{\jmath}$ & $10^{\prime}$ & 0,70 & 0,10 \\
\hline $12^{\prime}$ & 2,48 & 0,29 & $12^{\prime}$ & 1,34 & 0,16 & $12^{\prime}$ & 0,81 & 0,11 \\
\hline $14^{\prime}$ & 2,77 & 0,25 & $14^{\prime}$ & 1,50 & 0,16 & $14^{\prime}$ & 0,92 & 0,11 \\
\hline $16^{\prime}$ & 3,02 & $0,2 \check{a}$ & $16^{\prime}$ & 1,64 & 0,14 & $16^{\prime}$ & 1,03 & 0,11 \\
\hline $18^{\prime}$ & 3,27 & $0,2 \check{z}$ & $18^{\prime}$ & 1,79 & 0,15 & $18^{\circ}$ & 1,15 & 0,12 \\
\hline $20^{\prime}$ & 3,60 & 0,23 & $20^{\prime}$ & 1,94 & 0,15 & $20^{\prime}$ & 1,28 & 0,13 \\
\hline
\end{tabular}




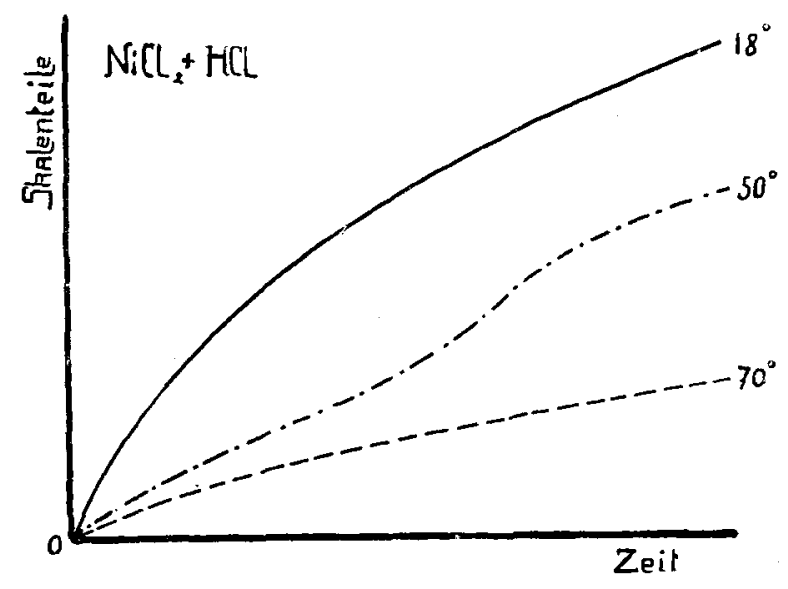

Fin: 1 .

Wie sich schon aus den Versuchen von Kohlschütter und Vuilleumis ergab, kann man im zeitlichen Gange der Kontraktion von Nickelniederschlägen aus verschiedenen Elektrolyten zwei Typen unterscheiden, insofern die Kontraktion bei den einen mit der Zeit nachlïsst und insgesamt kleinere Werte erreicht, bei anderen dagegen etwas zunimmt und bei gleicher Stromdauer auf einen höheren Gesamtbetrag kommt.

Im vorliegenden Falle verlangsamt sich die Kontraktion schon bei Zimmertemperatur und zwar in demselben Masse, wie der anfänglich unter dem Mikroskop vollständig strukturlos erscheinende Kathodenüberzug körniger wird. Mit steigender Temperatur ist der Verlauf der Kontraktion, wie aus den Kurven zu ersehen ist, dem Typus nach der gleiche, der nach gleichen Zeiten erreichte Effekt aber beträchtlich vermindert. Von Anfang an zeigte sich an den bei $50^{\circ}$ und $70^{\circ}$ hergestellten Niederschlägen ein deutlich körniges Gefüge, das nach Beendigung des Versuches schon mit blossem Auge zu erkennen war, während unter dem Mikroskop deutlich die einzelnen Krystallindividuen hervortraten. 


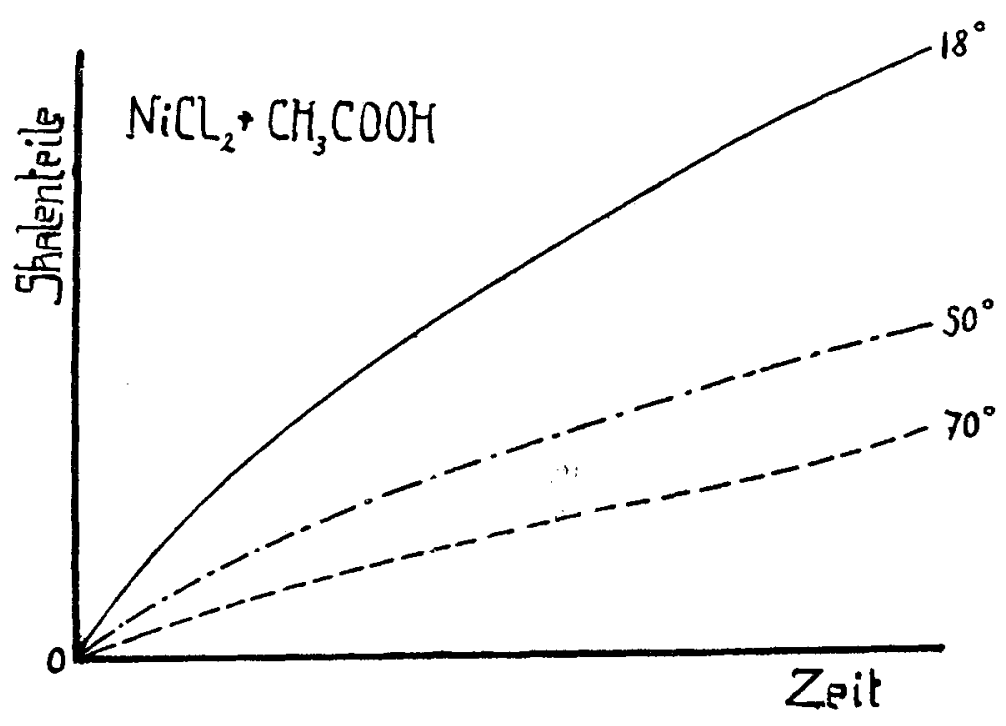

Fig. 2.

Die Ausbuchtung der Kurve für $50^{\circ}$ bei der salzsauren Lösung (Fig. 1) darf nicht ohne weiteres auf eine Unvollkommenheit der Versuchsausführung geschoben werden, sondern entspricht sehr wahrscheinlich einem gemässigten "Kontraktionsverzug", wie er in der Einleitung erwähnt und bei einer gleich zusammengesetzten Lösung bei $60^{\circ}$ früher viel ausgesprochener beobachtet wurde (vgl. Fig. 87 a. a. O.).

b) Versuche in n-NiCl${ }_{2}-$ Lösungen mit Ammoniumchloridund Borsäurezusatz.

Die Beobachtungen für diese Gruppe sind in Tab. III und IV und Fig. 3 und 4 zusammengefasst; es wurde hier auch noch der Verlauf für $60^{\circ}$ aufgenommen. 


\section{- $593-$}

Tabelle III.

n. $\mathrm{NiCl}_{2}, 0,5 \%$ Horsäure. Kathode II. $\mathrm{D}_{\mathrm{K}}=\tilde{5}$ Milliamp/(cm

\begin{tabular}{|c|c|c|c|c|c|c|c|c|}
\hline \multicolumn{3}{|c|}{ Nr. 17} & \multicolumn{2}{|c|}{ Nr. 20} & \multicolumn{2}{|c|}{ Nr. 22} & \multicolumn{2}{|c|}{$\mathrm{Nr} \cdot 23$} \\
\hline \multicolumn{3}{|c|}{$18^{\circ}$} & \multicolumn{2}{|c|}{$50^{\circ}$} & \multicolumn{2}{|c|}{$60^{\circ}$} & \multicolumn{2}{|c|}{$70^{\circ}$} \\
\hline $0^{\prime}$ & 0,00 & $\operatorname{Diff} / 2^{\prime}$ & 0,00 & $\operatorname{Diff} / 2^{\prime}$ & 0,00 & $\operatorname{Difl} / 2^{\prime}$ & 0,00 & $\operatorname{Diff} / 2^{\prime}$ \\
\hline $2^{\prime}$ & 0,81 & 0,81 & 0,55 & 0,55 & 0,35 & 0,35 & 0,24 & 0,24 \\
\hline $4^{\prime}$ & 1,53 & 0,72 & 0,99 & 0,44 & 0,66 & 0,31 & 0,43 & 0,19 \\
\hline $6^{\prime}$ & 2,48 & 0,95 & 1,32 & 0,43 & 0,93 & 0,27 & 0,63 & 0,20 \\
\hline $8^{\prime}$ & 3,38 & 0,90 & 1,82 & 0,50 & 1,20 & 0,27 & 0,84 & 0,21 \\
\hline $10^{\prime}$ & 4,52 & 1,14 & 2,20 & 0,38 & 1,47 & 0,27 & 1,40 & 0,20 \\
\hline $12^{\prime}$ & 5,57 & 1,05 & 2,50 & 0,39 & 1,71 & 0,24 & 1,57 & 0,17 \\
\hline $14^{\prime}$ & 6,58 & 1,01 & 2,88 & 0,29 & 2,00 & 0,29 & 1,76 & 0,19 \\
\hline $16^{\prime}$ & 7,82 & 1,24 & 3,26 & 0,38 & 2,25 & 0,25 & 1,96 & 0,20 \\
\hline $18^{\circ}$ & 9,03 & 1,21 & 3,51 & 0,25 & 2,52 & 0,27 & 2,13 & 0,17 \\
\hline $20^{\prime}$ & 10,18 & 1,15 & 3,88 & 0,47 & 2,76 & 0,24 & 2,33 & 0,20 \\
\hline
\end{tabular}

Tabelle IV.

n. $\mathrm{NiCl}_{2}$, n. $\mathrm{NH}_{4} \mathrm{Cl}$. Kathode $11 . \quad \Gamma_{\mathrm{K}}=5 \mathrm{Milliamp} / \mathrm{en}^{2}$

\begin{tabular}{|c|c|c|c|c|c|c|c|c|}
\hline \multicolumn{3}{|c|}{ Nr. 25} & \multicolumn{2}{|c|}{ Nr. 28} & \multicolumn{2}{|c|}{ Nr. 30} & \multicolumn{2}{|c|}{ Nr. 32} \\
\hline \multicolumn{3}{|c|}{$18^{0}$} & \multicolumn{2}{|c|}{500} & \multicolumn{2}{|c|}{$60^{\circ}$} & \multicolumn{2}{|c|}{$70^{\circ}$} \\
\hline $0^{\prime}$ & 0,00 & Diff $/ 2^{\prime}$ & 0,00 & $\operatorname{Diff} / 2^{\prime}$ & 0,00 & Dift $/ 2^{\prime}$ & 0,00 & 1)ifi/ $/ 2^{\prime}$ \\
\hline $2^{\prime}$ & 1,23 & 1,23 & 0,99 & 0,99 & 0,70 & 0,70 & 0,50 & 0,50 \\
\hline $4^{\prime}$ & 2,02 & 0,79 & 1,61 & 0,62 & 1,13 & 0,43 & 0,85 & 0,35 \\
\hline $6^{\prime}$ & 4,10 & 2,08 & 2,13 & 0,52 & 1,58 & 0,45 & 1,30 & 0,45 \\
\hline $8^{\prime}$ & 6,35 & 2,25 & 2,60 & 0,47 & 2,00 & 0,42 & 1,65 & 0,35 \\
\hline $10^{\prime}$ & 8,62 & 2,27 & 3,03 & 0,43 & 2,38 & 0,38 & 2,05 & 0,40 \\
\hline $12^{\prime}$ & 10,92 & 2,30 & 3,49 & 0,46 & 2,73 & 0,35 & 2,55 & 0,50 \\
\hline $14^{\prime}$ & - & - & 3,90 & 0,41 & 3,09 & 0,36 & 2,85 & 0,30 \\
\hline $16^{\prime}$ & - & - & 4,30 & 0,40 & 3,38 & 0,29 & 3,25 & 0,40 \\
\hline $18^{\prime}$ & - & - & 4,75 & 0,45 & 3,70 & 0,32 & 3,65 & 0,40 \\
\hline $20^{\prime}$ & - & - & 5,09 & 0,34 & 4,00 & 0,30 & 3,95 & 0,30 \\
\hline
\end{tabular}




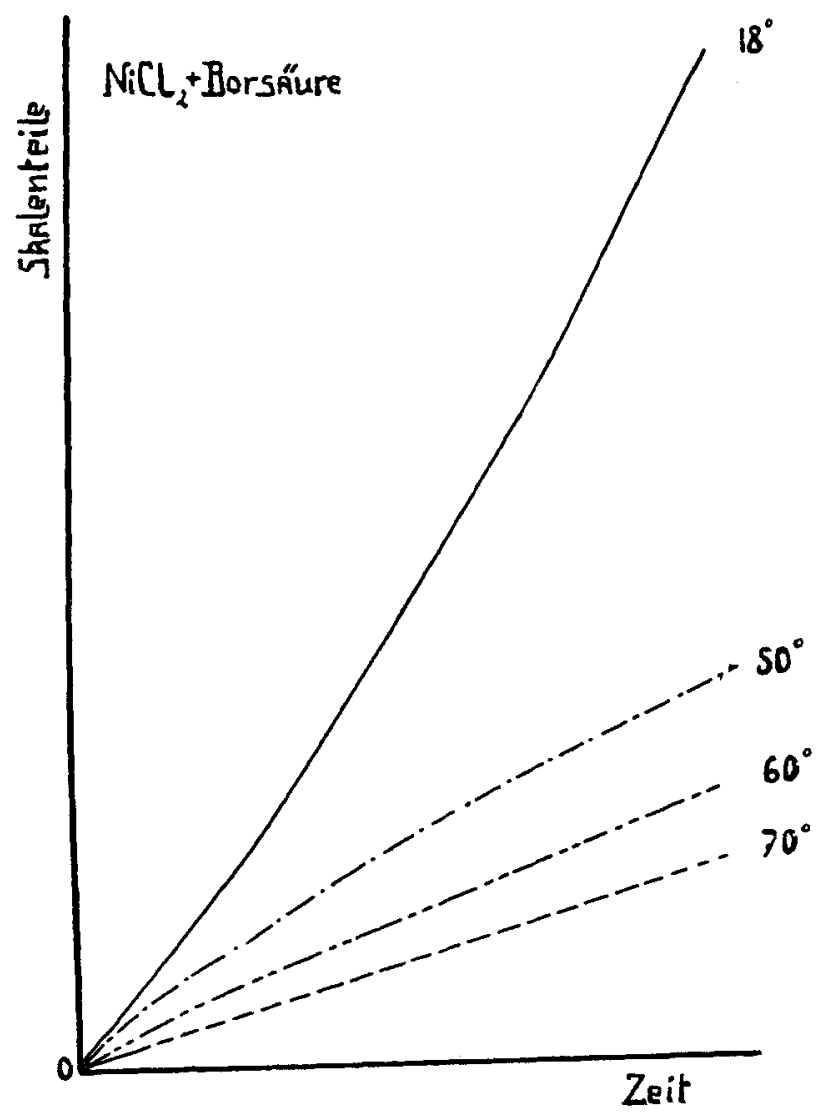

Fig. 3.

Die Kontraktion war in diesen Lösungen bei Zimmertemperatur bedeutend grösser als bei der Gruppe a und nahm mit der Zeit etwas zu, sodass sie nur während 10-12' beobachtet werden konnte, da der Zeiger dann schon den Elektrodenhalter berührte. Es wurden rein metallische aber braunstichige Ausscheidungen erhalten, deren Farbe schon auf ihre hochdisperse Struktur hinwies, und die beobachtete Veränderungsfähigkeit entsprach dieser Beschaffenheit. Bei den höheren Temperaturen nahm die Kontrahierbarkeit noch bedeutend stärker als in der Gruppe a ab, die Zeitkurven biegen sich ausserdem nach der Abszisse herab. Dem hierin sich äussernden Verhalten ging wiederum eine durch- 


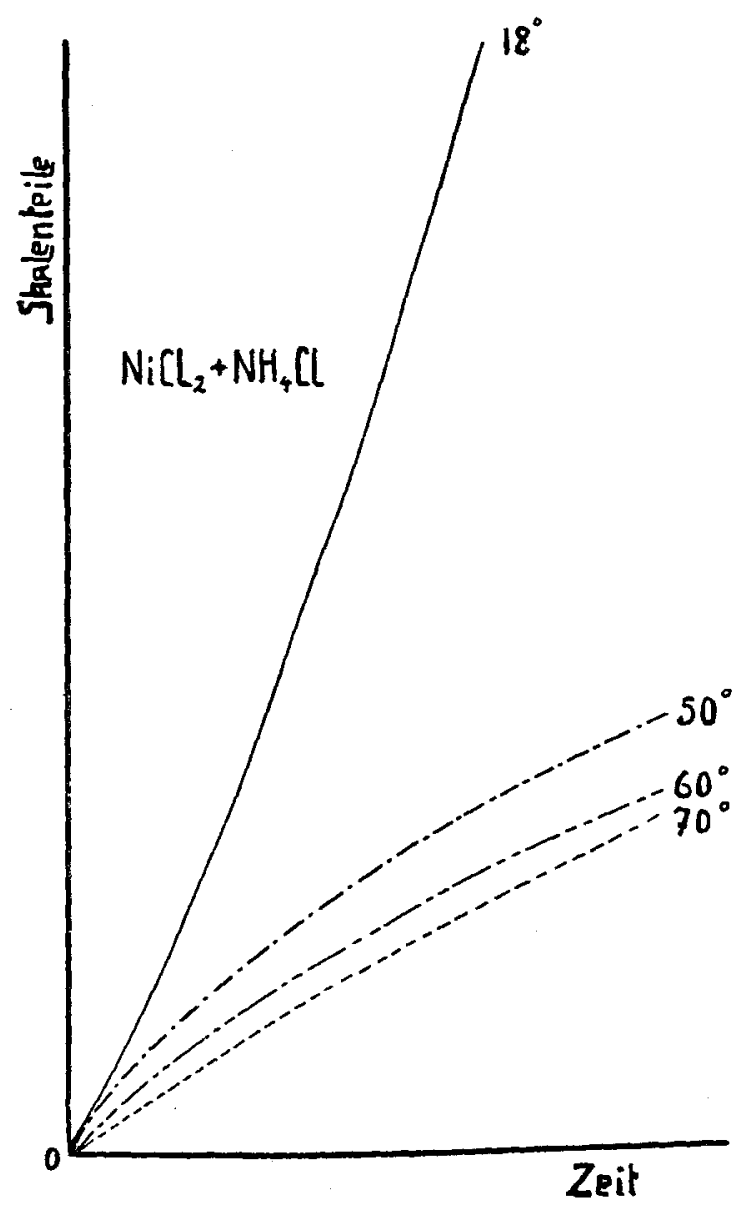

Fig. 4,

greifende Änderung der Struktur der Niederschläge parallel, indem an die Stelle der glatten braunstichigen Schichten, die bei gewöhnlicher Temperatur entstanden und mikroskopisch nicht auflösbar waren, deutlich körnige Überzüge traten, deren mikroskopisches Bild dem der Niederschläge aus der vorigen Gruppe ähnelte.

Zusammenfassend kann also festgestellt werden, dass der erwartete Einfluss der Bildungstemperatur auf die Struktur der Schichten und damit ihr mechanisches Verhalten besteht. Da die 
Ausbildung einer Gasschicht an der Elektrode durch Erhöhung der Temperatur erschwert werden muss, kann die verringerte Dispersität und ihre hierdurch verminderte Sinterungsfähigkeit sehr wohl auf eine Verminderung der Wasserstoffbeladung als Ursache zurückgeführt werden, wenn auch noch andere Erklärungen möglich sind.

\section{B. Versuche mit Depolarisatoren.}

Wenn die hochdisperse Beschaffenheit der Nickelschicht, die als Ursache ihrer Kontraktion und damit auch des Abbliatterns des Elektrolytnickels betrachtet wird, die Folge einer als Dispersionsmittel wirkenden Wasserstoffhaut ist, so war anzunehmen, dass Depolarisatoren, die diese Haut beseitigen, auch die Abscheidungsform des Metalls beeinflussen und den Kontraktionseffekt mehr oder minder vollständig aufheben würden.

Die Möglichkeit einer derartigen Wirkung von depolarisierenden Agentien wurde schon von Riedel diskutiert, der die Bildung eines unbeständigen Hydrides im Sinne Foersters als Ursache des Blätterns in Betracht gezogen und auch Versuche in dieser Richtung angestellt hat. Es gelang Riedel jedoch nicht, das Blättern einzuschränken, sodass er den Schluss zog, der Zerfall einer Wasserstoffverbindung oder -legierung könne nicht der Grund der eigentümlichen Erscheinung sein. Als Depolarisatoren verwendete er einerseits direkte Oxydationsmittel, wie Kaliumchlorat, Titansulfat, Eisenchlorid $u$, a., anderseits wasserstoffaddierende Substanzen wie Nitrobenzol, Terpentin und Acetylen.

Um die Verbindung mit diesen Versuchen zu bekommen, wurden teilweise dieselben Substanzen, jedenfalls Stoffe beider Art auch für die folgenden Versuche benutzt. Als Grundlage diente die mit Ammoniumchlorid versetzte Lösung, da diese den stärksten Effekt gibt und keine sichtbare Wasserstoffentwicklung in ihr auftritt, die Verdichtung zu einer Gasschicht also vermutlich besonders stark ist. Es war daher wahrscheinlich, dass hier auch eine Beeinflussung am ehesten zu erreichen und am leichtesten sichtbar zu machen sein werde.

a) Versuche mit Wasserstoffperoxyd.

Die Badflüssigkeit, wurde durch Zusatz von Perhydrol auf einen $\mathrm{H}_{2} \mathrm{O}_{2}$-Gehalt von 0,3 bezw. $1 \%$ gebracht, nachdem sich 
durch Vorversuche die verwendbaren Konzentrationen als in diese Grenzen fallend erwiesen hatten.

Bei einer Stromdichte von $5 \mathrm{MA} / \mathrm{cm}^{2}$ wurde in der Lösung mit $1 \%$ Wasserstoffperoxyd selbst bei einstündiger Dauer der Elektrolyse überhaupt kein Metall ausgeschieden, während in der mit $0,3 \%$ dann erst ein ganz geringer, die Kathode nicht gleichmässig bedeckender Niederschlag auftrat; dementsprechend bewegte sich auch der Zeiger nicht von seiner Anfangsstellung. Bei Steigerung der Stromdichte auf $10 \mathrm{MA} / \mathrm{cm}^{2}$ wurde in beiden Lösungen eine Ausscheidung von dunkelgrauer Farbe erhalten, die unter dem Mikroskop eine feinkörnige Struktur erkennen liess. Die Kontraktion verringerte sich beträchtlich und zunehmend mit dem Gehalt an Peroxyd, sodass sie sich 10 bezw. 18 Minuten lang beobachten liess, während in dem Grundelektrolyten ohne depolarisierenden Zusatz der Messbereich bereits nach 6 Minuten durchlaufen war. (Siehe Tab. V.) Gleichzeitig trat, wie aus der Fig. 5, in der auch der Verlauf bei $5 \mathrm{MA} / \mathrm{cm}^{2}$ mitaufgezeichnet ist, ersichtlich wieder die Verlangsamung ein, die auch bei höheren Temperaturen beobachtet wurde, sodass die beim reinen Elektrolyten beschleunigt ansteigenden Kurven in solche, die gegen die Abszisse geneigt sind, übergehen. Immerhin lagen noch beträchtlich sinterungsfähige, also feinteilige Niederschläge vor, die erst bei Fortsetzung der Elektrolyse über eine Stunde deutlich körnig wurden und hellgraue Farbe annahmen; sie lösten sich dann im Gegensatz zu dem im depolarisatorfreien Bade erhaltenen auffallend schwer in Säuren.

Tabelle V.

n. $\mathrm{NiCl}_{2}, \mathrm{n} . \mathrm{NH}_{4} \mathrm{Cl}$. - Wasserstoffperoxyd. Kathode $1 . \quad \mathrm{D}_{\mathrm{K}}=10 \mathrm{Nilliamp} / \mathrm{cm}^{2}$

\begin{tabular}{|c|c|c|c|c|c|c|c|c|}
\hline \multicolumn{3}{|c|}{ Nr. 47} & \multicolumn{3}{|c|}{ Nr. 49} & \multicolumn{3}{|c|}{ Nr. 54} \\
\hline \multicolumn{3}{|c|}{ Ohne Zusatz } & \multicolumn{3}{|c|}{$1 \%$ Zusatz } & \multicolumn{3}{|c|}{$0,3^{\circ} / 0$ Zusatz } \\
\hline $0^{\prime}$ & 0,00 & Dill $/ 2^{\prime}$ & $0^{\prime}$ & 0,00 & Difr/3' & $0^{\prime}$ & 0,00 & $\operatorname{DifT} 2^{\prime}$ \\
\hline $2^{\prime}$ & 3,00 & 3,00 & $3^{\prime}$ & 1,92 & 1,92 & $Q^{\prime}$ & 1,80 & 1,80 \\
\hline $4^{\prime}$ & 6,20 & 3,20 & $6^{\prime}$ & 3,14 & 1,22 & $4^{\prime}$ & 3,15 & 1,35 \\
\hline \multirow[t]{4}{*}{$6^{\prime}$} & 8,90 & 2,70 & $9^{\prime}$ & 4,14 & $1,(10$ & $6^{\prime}$ & 4,12 & 0,97 \\
\hline & & & $12^{\prime}$ & 5,22 & 1,08 & $8^{\prime}$ & 5,10 & 0,89 \\
\hline & & & $15^{\prime}$ & 6,52 & 1,30 & $10^{\prime}$ & $6,0.5$ & 0,95 \\
\hline & & & $18^{\prime}$ & 8,22 & 1,70 & & & \\
\hline
\end{tabular}






Fig. 5.

b) Versuche mit Nitrobenzol.

Die Nickellösung wurde kräftig mit Nitrobenzol durchgeschüttelt, mehrere Stunden stehen gelassen, damit sich feinverteilte Tröpfchen absetzen konnten, und mit einem kleinen Bodenbelag der schwerlöslichen Flüssigkeit verwendet.

Eine Metallabscheidung trat auch bei schwachen Strömen ein, und die Kontraktion nahm mit abnehmender Stromdichte stark ab. Während aber bei den Versuchen mit Wasserstoffperoxyd, sobald eine Beeinflussung der Abscheidungsform konstatierbar war, der Effekt sich mit der Zeit verringerte, ist hier der an- 
steigende Typus der Kurven (Fig. 6) bei den höheren Stromdichten noch deutlich erhalten. Dagegen waren die Niederschläge in allen Fällen fast schwarz gefärbt und hatten eine starke Neigung,



Fig. 6.

sich von der Unterlage abzulösen und zu blättern. Anscheinend wurde Nitrobenzol an der Kathode stark adsorbiert oder kataphoretisch dahin übergeführt, sodass es mit als Dispersionsmittel wirkte. Jedenfalls hat man es mit einer noch feineren Verteilungsform des Nickels zu tun, wie sich auch daraus ergab, dass sich das Metall sehr leicht in Säuren löste.

Wenn Nitrobenzol in Form einer verdiunnten alkoholischen Lösung zugesetzt wurde, war der Kontraktionsverlauf der gleiche, nur war der Niederschlag heller gefärbt und zeigte unter dem Mikroskop eine allerdings kaum erkennbare Krystallitbildung. 
Tabelle VI.

n. $\mathrm{NiCl}_{2}$, I. $\mathrm{NH}_{4} \mathrm{Cl}$. - Nitrobenzol. Kathode I

\begin{tabular}{|c|c|c|c|c|c|c|c|c|c|}
\hline \multicolumn{3}{|c|}{$\mathrm{Ne} .56$} & Nr. 58 & \multicolumn{2}{|c|}{ Nr. 59} & \multicolumn{2}{|c|}{ Nr. 61} & \multicolumn{2}{|c|}{ Nr. 63} \\
\hline \multicolumn{3}{|c|}{$\begin{array}{c}\text { Ohne Zusat: } \\
\mathrm{D}=5 \mathrm{Milliamp} / \mathrm{cm}^{2}\end{array}$} & $\begin{array}{c}\text { Mit Zusatz } \\
\mathrm{D}=5 \mathrm{M} \text {. A. } / \mathrm{cm}^{2}\end{array}$ & \multicolumn{2}{|c|}{7,5 M. A. $/ \mathrm{cm}^{2}$} & \multicolumn{2}{|c|}{8,3 N. A. $/ \mathrm{cm}^{2}$} & \multicolumn{2}{|c|}{$10 \mathrm{M} . \mathrm{A} / \mathrm{cm}^{2}$} \\
\hline $0^{\prime}$ & 0,00 & $\operatorname{Diff} / 2^{\prime}$ & $0,00-0,21$ in & 0,00 & $\operatorname{Diff} / 2^{\prime}$ & 0,00 & Diff $/ 2^{\prime}$ & 0,00 & Diff/2' \\
\hline $2^{\prime}$ & 1,38 & 1,38 & 20 Minuten & 0,15 & 0,15 & 0,31 & 0,31 & 0,35 & 0,35 \\
\hline $4^{\prime}$ & 3,17 & 1,79 & Niederschlag & 0,48 & 0,33 & 0,63 & 0,32 & 0,84 & 0,49 \\
\hline $6^{\prime}$ & 5.22 & 2,05 & $\begin{array}{l}\text { dunkel } \\
\text { schwarz }\end{array}$ & 0,81 & 0,33 & 1,05 & 0,42 & 1,44 & 0,60 \\
\hline $8^{\prime}$ & 7,47 & 2,25 & & 1,19 & 0,38 & 1,49 & 0,44 & 2,14 & 0,77 \\
\hline $10^{\prime}$ & 9,74 & 2,27 & & 1,60 & 0,41 & 1,90 & 0,46 & 2,82 & 0,68 \\
\hline $12^{\prime}$ & & & & 1,96 & 0,36 & 2,43 & 0,48 & 3,41 & 0,59 \\
\hline $14^{\prime}$ & & & & 2,36 & 0,40 & 2,96 & 0,53 & 4,09 & 0.68 \\
\hline $16^{\prime}$ & & & & 2,71 & 0,35 & 3,43 & 0,47 & 4,61 & 0,52 \\
\hline $18^{\prime}$ & & & & 3,06 & 0,35 & 3,98 & 0,55 & 5,30 & 0,69 \\
\hline $20^{\prime}$ & & & & 3,43 & 0,37 & 4,48 & 0,50 & 6,00 & 0,70 \\
\hline
\end{tabular}

c) Versuche mit Kalinsmchlorat.

Ähnlich wie bei den Versuchen mit Wasserstoffperoxyd liess sich auch hier eine Strukturbeeinflussung der Niederschiäge durch den Depolarisator nur innerhalb enger, obendrein von der Stromdichte stark abhängiger Konzentrationsgrenzen verfolgen.

In Lösungen, die 0,5-normal für $\mathrm{KClO}_{3}$ waren, trat erst bei einer Stromdichte von $20 \mathrm{M}$. A. $/ \mathrm{cm}^{2}$ Nickelabscheidung ein; die Ausbeute betrug nach einstündiger Elektrolyse nur 3,4\%.

Mit abnehmender Konzentration an Kaliumchlorat rückte die Stromdichte, mit welcher Metallausscheidung zu erreichen war, herab, und die Ausbeute verbesserte sich, sodass sie bei 0,25-n. $\mathrm{KClO}_{3}$ mit 10 M. A. $/ \mathrm{cm}^{2} \quad 7,1 \%$ mit 20 M. A. $/ \mathrm{cm}^{2} \quad 7,5 \%$, bei 0,125-n. $\mathrm{KClO}_{3}$ mit $5,10,20 \mathrm{M}$. A./ $\mathrm{cm}^{2}$ nach einstündiger Elektrolyse $10,2,11,6,11 \%$ betrug. Die Kontraktometerausschläge waren, den geringen Niederschlagsmengen entsprechend, gering; die Niederschläge selbst zeigten unter dem Mikroskop ein feinkörniges Gefüge.

In $\mathrm{n} / 16$ - und $\mathrm{n} / 32-\mathrm{KClO}_{3}$-lösungen waren normale Beobachtungen möglich; die Resultate sind in Tab. VII enthalten; Fig. 7 gestattet den Einfluss der Stromdichte und des Depolarisators in seinen charakteristischen Zügen zu übersehen. 


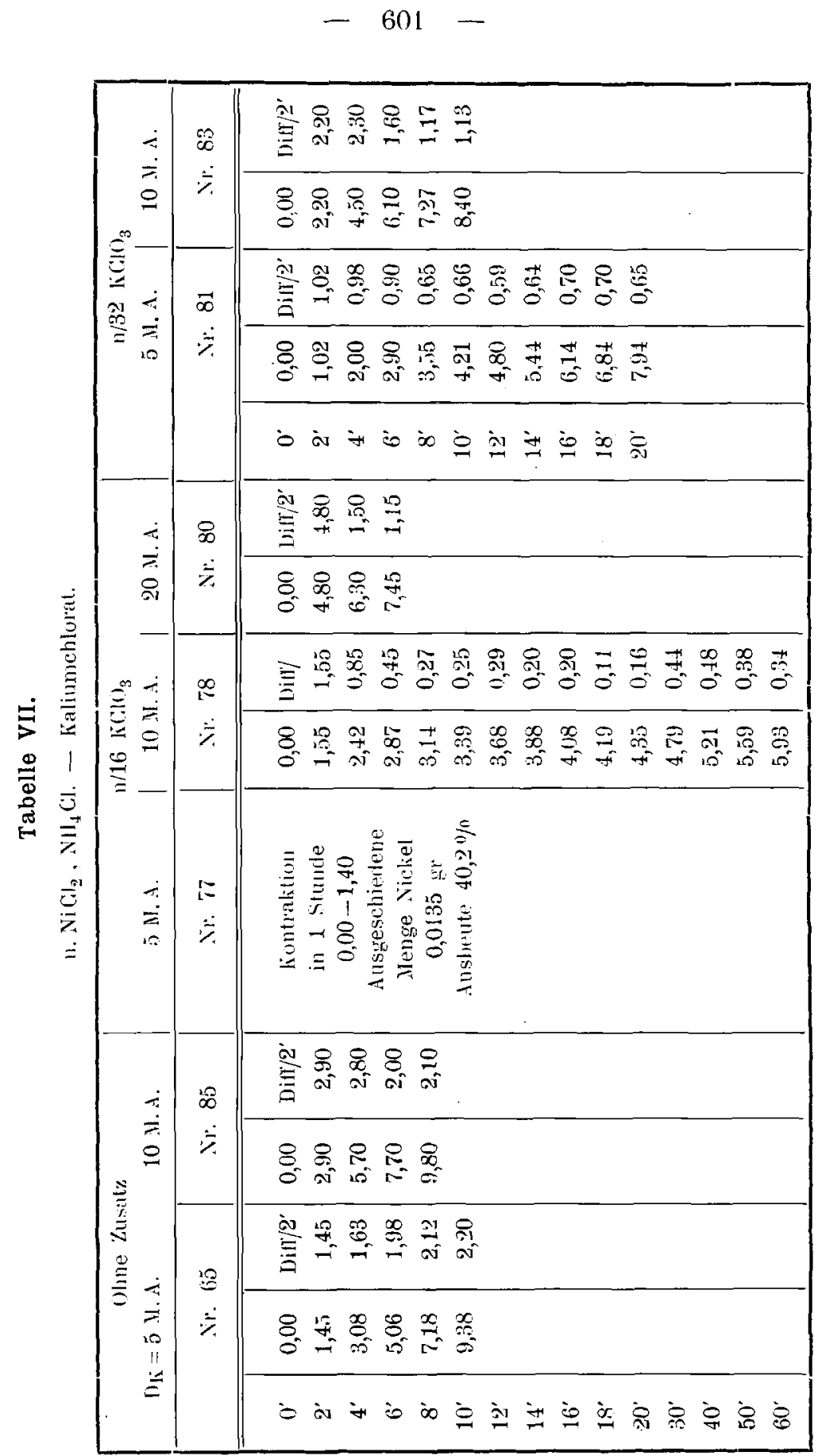




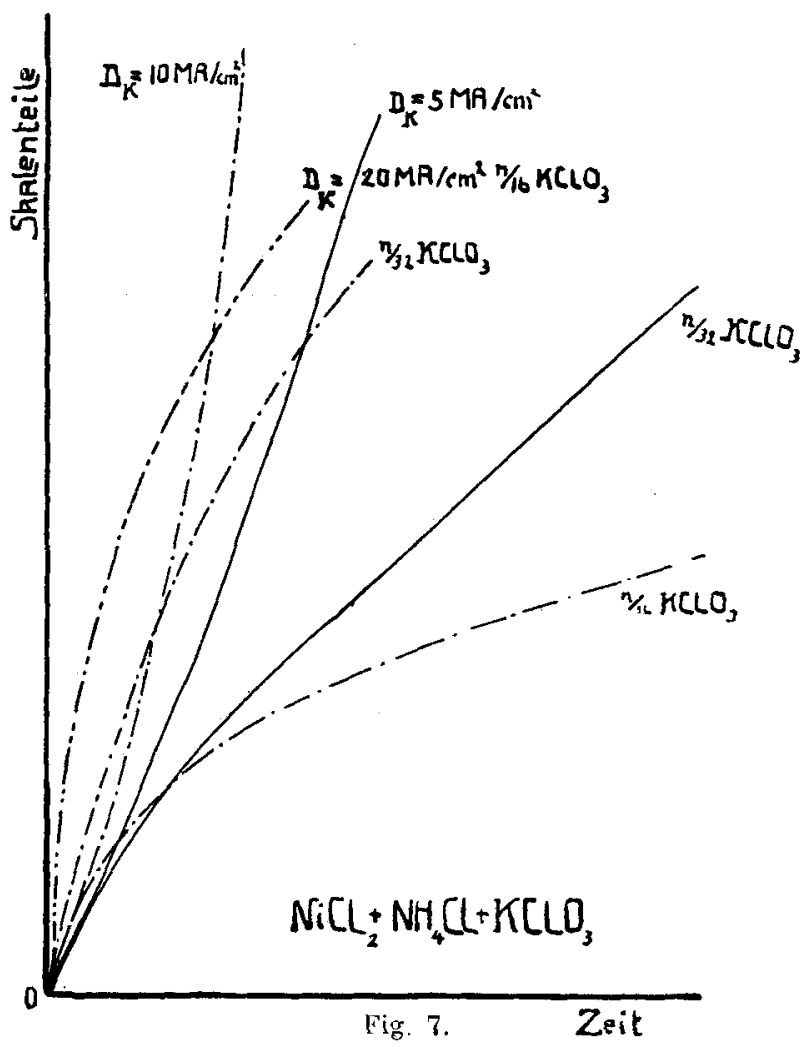

Die durch die Wirkung des Oxydationsmittels veränderte Beschaffenheit der Niederschläge tritt in der Gestalt der Kontraktionskurven deutlich hervor; im Aussehen war noch keine Veränderung zu erkennen; die Überzüge waren noch braunstichig in der Farbe und ohne auflösbare Struktur.

d) Versuche mit zimtsaurem Natrium.

In der Erwartung, dass der auf die Wirkung einer Wasserstoffhaut zurïckgefuihrte Effekt sich auch durch eine zur Wasserstoffaddition befähigte Substanz unterdrücken lassen und eine solche daher dieselbe Strukturänderung wie ein Oxydationsmittel herbeifuihren werde, sollte noch ein Versuch mit einer ungesättigten Kohlenstoffverbindung gemacht werden. Auf der Suche nach einer geeigneten Substanz, von der keine sonstigen Komplikationen zu befürchten waren, fiel die Wahl auf zimtsaures Natrium, das zwar wenig, aber hinreichend löslich ist, und in dem Nickelbade mit einem kleinen Bodenkörper zur Anwendung kam. 
Nach Stromschluss trat an der Kathode während etwa einer Minute starke Gasentwicklung auf, die in allen anderen Fällen nie beobachtet wurde.

Tabelle VIII.

n. $\mathrm{NiCl}_{3}, \mathrm{NH}_{4} \mathrm{Cl} .-\mathrm{C}_{6} \mathrm{H}_{5} \cdot \mathrm{CH}=\mathrm{CH} \cdot \mathrm{COONa} . \quad \mathrm{D}=5 \mathrm{Milliamp} / \mathrm{cm}^{2}$

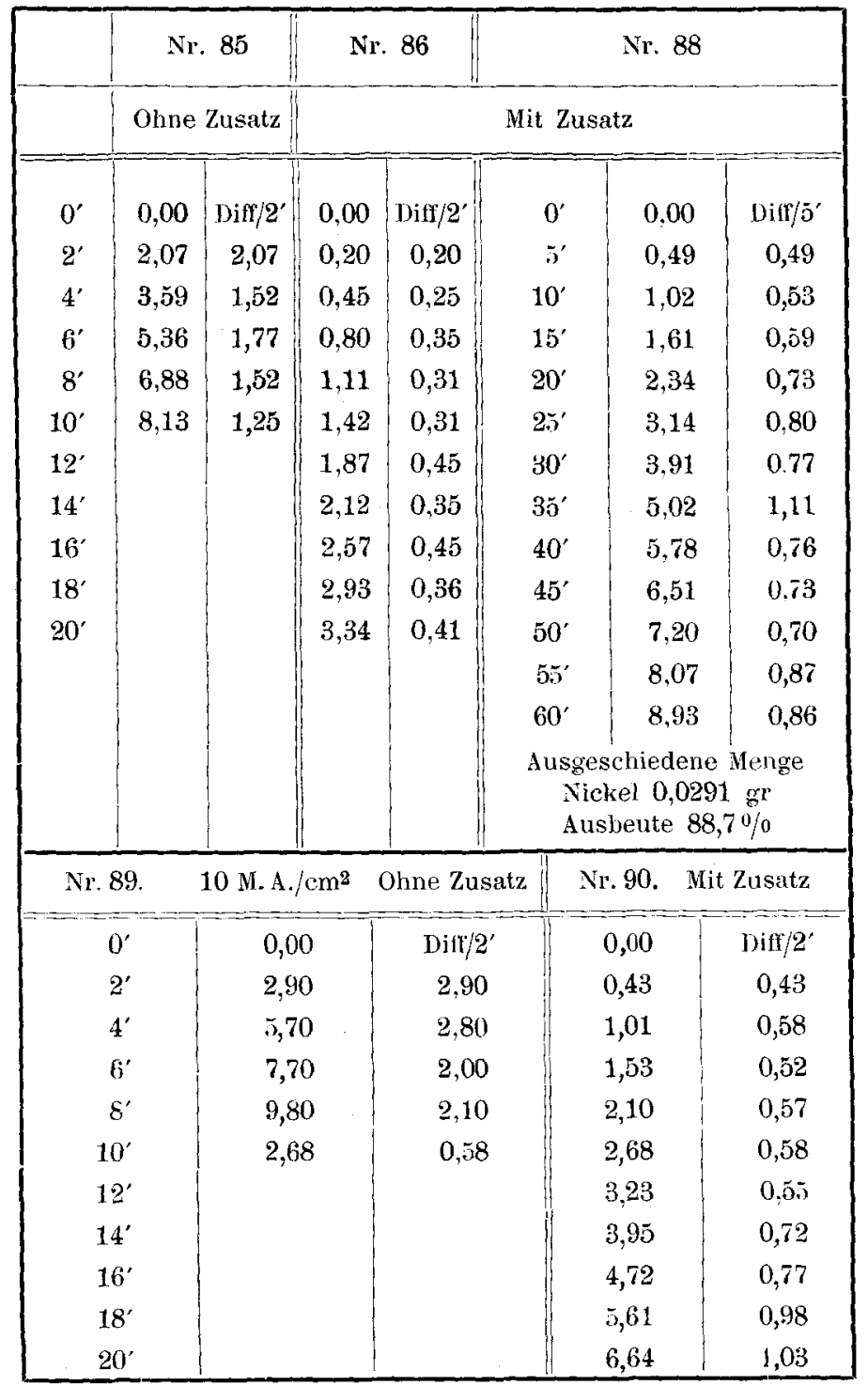


Die Niederschläge wiesen den typischen Übergang von der' braunstichigen, strukturlosen zur grauen, metallisch glänzenden Form auf und zeigten unter dem Mikroskop deutlich krystalline Beschaffenheit. Die Verminderung der Kontraktion gegenüber der im Grundelektrolyten war sehr bedeutend (Tab. VIII), der Verlauf derselben aber vom gleichen Typus, wie in jenem (Fig. 8).

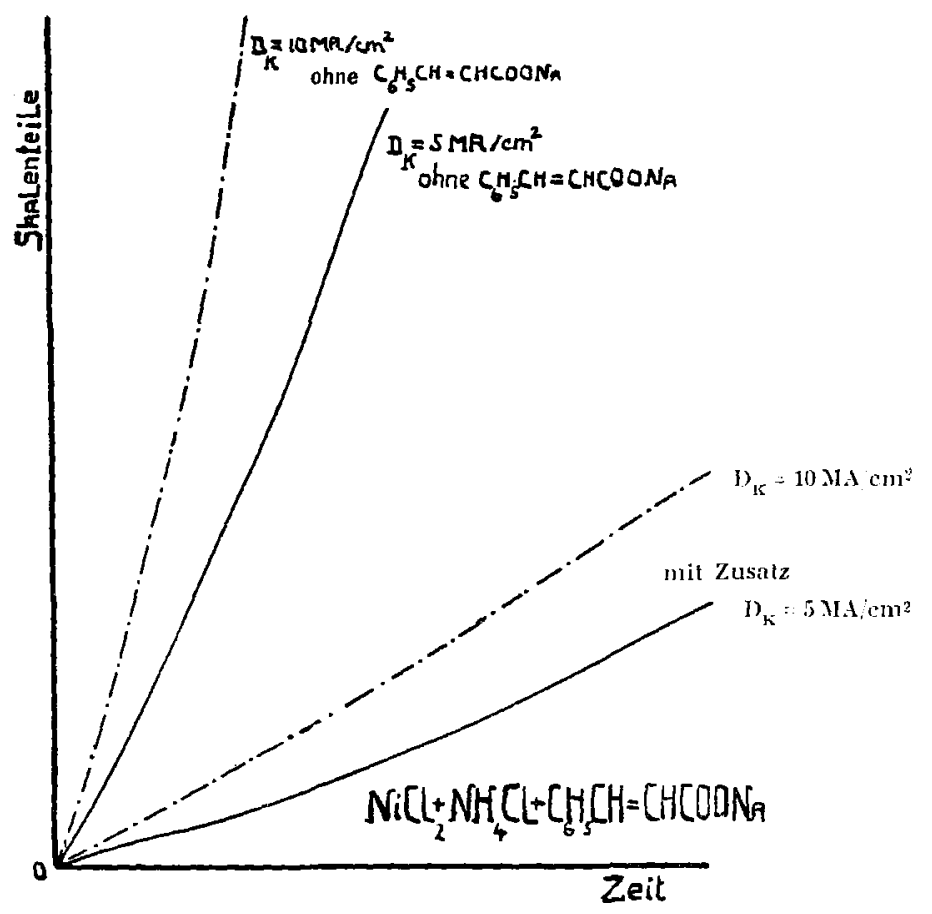

Fig. 8.

C. Versuche mit überlagertem Wechselstrom.

Bei der Anwendung verschiedener Wasserstoffdepolarisatoren wurde in den meisten Fällen eine Beeinflussung der Nickelniederschläge sowohl in ihrem unmittelbaren Aussehen, wie namentlich in ihrer inneren, in der spontanen Kontraktionsfähigkeit sich verratenden Struktur festgestellt, die ganz in der erwarteten Richtung lag. Es gelang jedoch nicht, die Veränderungsfähigkeit, die mit dem primären Auftreten einer Wasserstoffhaut an der Kathode in Zusammenhang gebracht wird, gänzlich zu unterdrücken, m. a. W. also die Ausbildung der Gasschicht vollständig zu ver- 
hindern. Wo sich ein Oxydationsmittel an sich als wirksam erwies, hatte die Steigerung seiner Konzentration mehrfach eine starke Herabsetzung der Stromausbeute oder sogar das Ausbleiben einer Metallabscheidung überhaupt zur Folge, es war also nicht möglich, die Oxydationswirkung so abzustufen, dass sie nur den Wasserstoff, nicht aber zugleich auch das Metall erfasste. Ausserdem hat die Anwendung von chemischen Oxydationsmitteln den Nachteil, dass fremde Substanzen in die Badflüssigkeit kommen, die in zunächst unübersehbarer Weise auch den Niederschlag beeinflussen können.

Letzteres musste sich nun vermeiden lassen, wenn man statt besonderer stofflicher Träger der Oxydationswirkung einen anderen, den gleichen Effekt herbeiführenden Vorgang heranziehen konnte, und es erschien möglich, dies durch Überlagerung von Wechselstrom über den Vorgang an der Gleichstromkathode zu erreichen und damit zugleich eine zweckmässige Abstufung der Wirkung zu erzielen.

Überlagerung von Wechselstrom ist bei elektrolytischen Vorgängen schon mehrfach zur Anwendung gekommen.

So untersuchte Ruer ${ }^{1}$ ) die anodische Auflösung von Platin und Blei in Schwefelsäure bei gleichzeitiger Einwirkung von Gleichstrom und Wechselstrom und kam zu dem Schluss, dass der Wechselstrom das Auftreten höherer Oxyde verhindert oder Bedingungen für die Bildung niederer, löslicher Oxyde schafft, während Bennewitz ${ }^{2}$ ) fand, dass die Zersetzungsspannung von Wasser durch ïberlagerte elektrische Wellen herabgesetzt wird. Andererseits konstatierten von Wartenberg und Archibald ${ }^{3}$ ) eine erhebliche Beeinflussung der Ausbeute der anodischen Ozonbildung in Schwefelsäure durch Verstärkung des Wechselstromes bei der Elektrolyse mit asymmetrischen Wechselströmen, und Reitlinger ${ }^{4}$ ) verfolgte den Einfluss von Wechselstrom auf die Bildung verschiedener anodischer Oxydationsprodukte, indem er von dem Gedanken ausging, dass der überlagerte Wechselstrom die Überspannung zerstört und daher die Bildung desjenigen Körpers begünstigen muss, für dessen Bildung ein geringeres Oxydationspotential erforderlich ist. Schliesslich hat $G r u b e^{3}$ ), von ähnlichen Überlegungen geleitet, die Vorgänge bei der anodisehen Sauer-

1) Z. ph. Ch. 44, 81 (1903).

16, $812(1910)$.
4) Z. E.. Ch. 20, 261 (1914). 2) Z. ph. Ch. 72, $202(1910)$. 3) Z. Ll. Ch. 
stoffentwicklung an Platinanoden verfolgt, um das Auftreten verschiedener Oxydationsstufen des Platins durch schrittweise Reduktion mit der kathodischen Komponente des Wechselstroms im Potential hervortreten zu lassen, und neuerdings ${ }^{1}$ ) gezeigt dass die Stromausbeute an Natriumferrat durch Überlagerung von Wechselstrom über die Anode erheblich gesteigert werden kann.

In allen diesen Fällen handelt es sich um Beeinflussung von Anodenvorgängen, während die Einwirkung von Wechselstrom auf Kathodenvorgänge meines Wissens noch nicht untersucht wurde. Gerade die gute Dosierbarkeit der Depolarisation, die mit Wechselstrom erzielbar ist, empfahl aber seine Verwendung für meine Untersuchung.

In Ermangelung spezieller Hilfsmittel verwendete ich den gewöhnlichen städtischen Wechselstrom von 40 Perioden per Sekunde und 125 Volt Spannung, die durch einen kleinen Transformator auf 5 Volt heruntergebracht wurde. Die Zufuhr des Stromes zur Kathode geschah durch eine kleine an einen Draht angeschweisste Platinplatte, die gegen die Elektrode gebogen war. Um zu verhindern, dass die Zuleitung vom Gleichstrom als Zwischenleiter benutzt würde, wurde - in Anlehnung an die Anordnung Grube's - die ganze Wechselstromhülfselektrode in Glas eingeschmolzen und an ihr nur gegenuiber der Kathode eine Öffnung, die der Grösse der Platinplatte entsprach, freigelassen. Die Hülfselektrode wurde vor den Versuchen immer stark vernickelt.

Bei einigen qualitativen Vorversuchen ergab sich schon eine vollständige Strukturveränderung des Niederschlages unter der Wirkung des Wechselstromes. Unter dem Mikroskop beobachtete man bei den dünnsten Ausscheidungen ein stark schuppiges Aussehen, und auch die Farbe hatte sich verändert. Währenddem. man bei der gewöhnlichen Elektrolyse den braunstichigen Niederschlag erhielt, bekam man jetzt schöne graue, metallisch glänzende und fest haftende Überzüge, die Kontraktion aber war in allen Fällen bedeutend reduziert.

Wenn man den Gleichstrom zuerst allein fliessen liess und erst, wenn die Kontraktion im Gange war, den Wechselstrom überlagerte, beobachtete man sofort eine Verzögerung der Kontraktion, die' sich bei einer nachfolgenden wechselstromlosen Periode sprunghaft auszugleichen suchte.

1) Z. El. Ch. 26, 161 (1920). 
Wichtig erschien mir vor allem, das Verhältnis der Stromausbeuten unter diesen Bedingungen zu verfolgen, während gleichzeitig das Verhältnis der beiden Ströme variiert wurde. Es wurde daher noch ein Kupfercoulometer (mit Oettel'scher Lösung) in den Gleichstromkreis geschaltet.

Wie aus der Tabelle IX zu ersehen ist, wurde die Stromausbeute durch die Überlagerung von Wechselstrom stets herabgesetzt, in den salz- und essigsauren Lösungen verhältnismässig weniger als in denjenigen mit Borsäure- bezw. Ammoniumchloridzusatz.

Tabelle IX.

Stromausbeuten bei ïberlagertem Wechselstrom. Gleichstroindichte $5 \mathrm{M}$. A $/ \mathrm{cm}^{2}$

\begin{tabular}{|c|c|c|c|c|c|}
\hline $\begin{array}{l}\text { Vers. } \\
\text { Nr. }\end{array}$ & $\begin{array}{l}\text { Ver' } \\
\text { Bl'strom }\end{array}$ & $\begin{array}{l}\text { hältıis } \\
\text { : W'strom }\end{array}$ & $\begin{array}{c}\text { Ausgeschiedenes } \\
\text { Kupfer }\end{array}$ & $\begin{array}{c}\text { Ausgeschiedenes } \\
\text { Nickel }\end{array}$ & Ausbeute \\
\hline \multicolumn{6}{|c|}{ a) $0,25-n . \mathrm{NiCl}_{2}, 0,02-\mathrm{n} . \mathrm{HC}: \mathrm{l}$} \\
\hline 133 & ohne: & W'strom & $0,0344 \mathrm{gr}$ & $0,021 \mathrm{gr}$ & $66,25 \%$ \\
\hline 134 & 1 & $: 10$ & 0,0332 & 0,0198 & $64,61 \%$ \\
\hline 135 & 1 & $: \quad 20$ & 0,0317 & 0,0195 & $58,28 \%$ \\
\hline 136 & 1 & $: 40$ & 0,0398 & 0,0194 & $53,01 \%$ \\
\hline \multicolumn{6}{|c|}{ b) $0,25-\mathrm{n} . \mathrm{NiCl}_{2}, \mathrm{O} \cdot 1-\mathrm{n} \cdot \mathrm{CH}_{3} \cdot \mathrm{COOH}$} \\
\hline 129 & ohue & W'strom & $0,0248 \mathrm{gr}$ & $0,0142 \mathrm{gr}$ & $62,04 \%$ \\
\hline 130 & 1 & $: 10$ & 0,0236 , & $0,0134 \pi$ & $59,68 \% / 0$ \\
\hline 131 & 1 & $: \quad 20$ & $0,0432 \pi$ & $0,0234 \%$ & $58,56^{0}, 0$ \\
\hline 132 & 1 & $: 40$ & 0,0348 & 0,0172 & $53,56 \%$ \\
\hline \multicolumn{6}{|c|}{ c) n. $\mathrm{NiCl}_{2}, 0,5 \%$ Borsäure } \\
\hline 125 & ohne & W'strom & $0,0253 \mathrm{gr}$ & $0,0211 \mathrm{gr}$ & $90,12 \% / 0$ \\
\hline 126 & 1 & $: 10$ & 0,0390 & 0,0281 & $78,07 \% / 0$ \\
\hline 127 & 1 & $: 20$ & 0,0228 & 0,0158 & $75,09 \%$ \\
\hline 128 & 1 & $: 40$ & 0,0302 & 0,0200 & $71,76 \%$ \\
\hline \multicolumn{6}{|c|}{ n. $\mathrm{NiCl}_{2}, \mathrm{NH}_{4} \mathrm{Cl}$} \\
\hline 121 & ohne & W'strom & $0,0271 \mathrm{gr}$ & $0,0232 \mathrm{gr}$ & $92,40 \%$ \\
\hline 122 & 1 & $: 10$ & $0,03 \overline{3} n_{n}$ & 0,0285 & $79,39 \%$ \\
\hline 123 & 1 & $: 20$ & 0,0358 & 0,0260 & $78,69 \%$ \\
\hline 124 & 1 & : 40 & 0,0344 & 0,0214 & $67,40 \%$ \\
\hline
\end{tabular}


Versuche mit Nickelsulfatlïsungen.

Die vorausgehenden Versuche über die Kontraktion der Nickelniederschläge wurden mit Chloridlösungen und Nickelanoden ausgeführt, da unter diesen Bedingungen nicht nur die Konzentration der Lösungen konstant bleibt, sondern auch die Klemmenspannung wegen der Angreifbarkeit der Anode sich nicht ändert.

Solange es sich nur um die Bildung des Nickelniederschlages unter normalen elektrolytischen Bedingungen handelte, war diese Anordnung die gegebene.

Um aber einheitlichere Verhältnisse für die Depolarisationswirkung durch Wechselstrom zu haben, schien es zweckmässig, einen sauerstoffentwickelnden Elektrolyten anzuwenden und demgemäss mit Sulfatlösungen zu arbeiten.

In solchen Lösungen sind Nickelanoden nur teilweise angreifbar. Es bilden sich zwar anodisch auch Nickelionen, doch überzieht sich die Elektrode zum Teil mit Nickeloxyd oder -Peroxyd, was ein Ansteigen der Klemmenspannung zur Folge hat, sodass die Stromstärke dann dauernd nachreguliert werden muss. Um die Bedingungen auch in dieser Beziehung einheitlich zu gestalten, wurde daher weiterhin mit Platinanoden gearbeitet.

Die Tabellen $\mathrm{X}$-XIII enthalten einige Versuche mit reinen Sulfatlösungen und solche mit Zusätzen. Aus ihnen ergibt sich, dass die Kontraktion bei Anwendung von Sulfat schon ohne Zuschaltung von Wechselstrom geringer ist, als unter entsprechenden Bedingungen bei Chlorid; der Wechselstrom setzt sie in ähnlichem Verhältnis wie bei diesen herab; bei dem Versuch mit Borsäure (Nr. 104) trat ein typischer Kontraktionsverzug auf, der mit Wechselstrom nicht beobachtet wurde. Ein Zusatz von Alkohol zur borsäurehaltigen Lösung drückte die Kontraktion etwas herab und schien auch den Einfluss des Wechselstroms zu verringern.

Das Metall scheidet sich aus den Sulfatlösungen von vornherein in etwas dichterer Form ab, als aus Chloridlösungen, die Wechselstromüberlagerung ruft aber die gleiche Strukturänderung hervor, wie in diesen. 
Tabelle X.

Neutrale $\mathrm{n}$-Nickelsulfatlösung. $\mathrm{D}_{\mathbf{K}}=\mathbf{5}$ Milliamp. $/ \mathrm{cm}^{2}$

\begin{tabular}{|r|c|r||r|r|}
\hline \multicolumn{3}{|c||}{ Nr. 92 } & \multicolumn{2}{c|}{ Nr. 95 } \\
\hline \multicolumn{2}{|c||}{ Ohne W'strom } & \multicolumn{2}{c|}{ Mit W'strom } \\
\hline \hline $0^{\prime}$ & 0,00 & Diff $/ 5^{\prime}$ & 0,00 & Diff $/ 5^{\prime}$ \\
$5^{\prime}$ & 0,41 & 0,41 & 0,38 & 0,38 \\
$10^{\prime}$ & 1,20 & 0,79 & 0,54 & 0,16 \\
$15^{\prime}$ & 1,80 & 0,60 & 0,67 & 0,13 \\
$20^{\prime}$ & 2,28 & 0,48 & 0,85 & 0,18 \\
\multicolumn{3}{|c|}{ Wechselstromdichte 50 Milliamp./cm² } \\
\hline
\end{tabular}

Tabelle XI.

0,25-n. Nickelsulfat, $0,1 \mathrm{n}$. Essigsäure. $\mathrm{D}_{\mathrm{K}}=5$ Milliamp. $/ \mathrm{cm}^{2}$

\begin{tabular}{|r|r|r|r|r|}
\hline \multicolumn{3}{|c||}{ Nr. 96} & \multicolumn{2}{c|}{ Nr. 101 } \\
\hline \multicolumn{3}{|c||}{ Ohne W'strom } & \multicolumn{2}{c|}{ Mit W'strom } \\
\hline $0^{\prime}$ & 0,00 & Diff $/ 5^{\prime}$ & 0,00 & Diff $/ 5^{\prime}$ \\
$15^{\prime}$ & 0,40 & 0,40 & 0,11 & 0,11 \\
$10^{\prime}$ & 0,68 & 0,28 & 0,29 & 0,18 \\
$15^{\prime}$ & 0,88 & 0,20 & 0,033 & 0,23 \\
$20^{\prime}$ & 1,07 & 0,10 & 0,72 & 0,19 \\
\multicolumn{3}{|c|}{ Wechselstromdichte 50 Nilliamp./cm2 } \\
\hline
\end{tabular}

Tabelle XII.

n. Nickelsulfat, $0,5 \%$ Borsäure. $D_{\mathrm{K}}=5$ Milliamp. $/ \mathrm{cm}^{3}$

\begin{tabular}{|c|c|c|c|c|}
\hline \multicolumn{3}{|c|}{ Nr. 104} & \multicolumn{2}{|c|}{ Nr. 107} \\
\hline & \multicolumn{2}{|c|}{ Ohne W'strom } & \multicolumn{2}{|c|}{ Mit W'strom } \\
\hline $0^{\prime}$ & 0,00 & $\operatorname{Difr} / 5^{\prime}$ & 0,00 & $\operatorname{Ditf} / 5^{\prime}$ \\
\hline $5^{\prime}$ & 0,18 & 0,18 & 0,27 & 0,27 \\
\hline $10^{\circ}$ & 0,52 & 0,34 & 0,72 & 0,45 \\
\hline $15^{\prime}$ & 0,74 & 0,22 & 1,44 & 0,72 \\
\hline $20^{\prime}$ & 1,40 & 0,66 & 2,19 & 0,75 \\
\hline $25^{\prime}$ & 2,07 & 0,76 & 2,81 & 0,65 \\
\hline $30^{\prime}$ & 8,50 & 6,43 & 3,39 & 0,55 \\
\hline \multicolumn{5}{|c|}{27 sprung $(2,2-8,2)$} \\
\hline \multicolumn{5}{|c|}{ Wechselstromdichte 50 Milliamp. $/ \mathrm{cm}^{2}$} \\
\hline
\end{tabular}




\section{$-610-$}

Tabelle XIII.

n. Nickelsulfat, $0,5 \%$ Borsäure $+25 \%$ Alkohol, $D_{K}=5$ Milliamp. $/ \mathrm{en}^{2}$

\begin{tabular}{|c|c|c|c|c|}
\hline \multicolumn{3}{|c|}{ Nr. 112} & \multicolumn{2}{|c|}{ Nr. 114} \\
\hline \multicolumn{3}{|c|}{ Ohne W'strom } & \multicolumn{2}{|c|}{ Mit W'strom } \\
\hline $0^{\prime}$ & 0,00 & Dift/ $/ 2^{\prime}$ & 0,00 & Difr/2' \\
\hline $2^{\prime}$ & $0,3 \mathbf{3}$ & 0,35 & 0,29 & 0,29 \\
\hline $4^{\prime}$ & 0,84 & 0,49 & 0,63 & 0,34 \\
\hline $6^{\prime}$ & 1,34 & 0.50 & 0,98 & 0,35 \\
\hline $8^{\prime}$ & 1,92 & 0,58 & 1,39 & 0,41 \\
\hline $10^{\prime}$ & 2,43 & $0, \check{1} 1$ & 1,82 & 0,43 \\
\hline $12^{\prime}$ & 2,95 & 0,52 & 2,22 & 0,40 \\
\hline $14^{\prime}$ & 3,54 & 0,59 & 2,62 & 0,40 \\
\hline $16^{\prime}$ & 4,12 & 0,58 & 3,04 & 0,42 \\
\hline $18^{\prime}$ & 4,71 & 0,59 & 3,44 & 0,40 \\
\hline $20^{\prime}$ & 5,23 & 0,52 & 3,88 & 0,44 \\
\hline \multicolumn{5}{|c|}{ Wechselstromaichte 50 Milliamp. $/ \mathrm{cm}^{2}$} \\
\hline
\end{tabular}

Einfluss der Wechselstromdichte auf die Struktur der Niederschläge.

Tabelle XIV.

n. Nickelsulfat $+0,5 \%$ Borsäure. $D_{K}=5$ Milliamp. $/ \mathrm{cm}^{2}$

\begin{tabular}{|c|c|c|c|c|c|c|c|c|c|c|}
\hline & \multicolumn{2}{|c|}{ Nr. 117} & \multicolumn{2}{|c|}{ Nr. 118} & \multicolumn{2}{|c|}{ Nr. 119} & \multicolumn{2}{|c|}{ Nr. 120} & \multicolumn{2}{|c|}{ Nr. 121} \\
\hline \multirow{2}{*}{\multicolumn{3}{|c|}{ Ohne W'strom }} & \multicolumn{2}{|c|}{ G.-St. : W.-St. } & \multicolumn{2}{|c|}{ G.-St. : W.-St. } & \multicolumn{2}{|c|}{ G.-St. : W.-St. } & \multicolumn{2}{|c|}{$\mathrm{G}_{1}-\mathrm{St}$. : W.-St. } \\
\hline & & & $1:$ & 0,5 & 1 & $: \quad 1$ & 1 & 2 & 1 & $\check{5}$ \\
\hline $0^{\prime}$ & 0,00 & Difl/2' & 0,00 & $\operatorname{Diff} / 2^{\prime}$ & 0,00 & Dify/2' & 0,00 & Diff $/ 2^{\prime}$ & 0,00 & Diff $/ 2^{\prime}$ \\
\hline $2^{\prime}$ & 0,70 & 0,70 & 0,80 & 0,80 & 0,70 & 0,70 & 0,60 & 0,60 & 0,60 & 0,60 \\
\hline $4^{\prime}$ & 1,43 & 0,73 & $1.8 \tilde{5}$ & 1,05 & 1,20 & 0,50 & 1,20 & 0,60 & 1,20 & 0,60 \\
\hline $6^{\prime}$ & 2,03 & 0,59 & 2,55 & 0,70 & 1.53 & 0,33 & $1, \check{0} 0$ & 0,30 & 1,60 & 0,40 \\
\hline $8^{\prime}$ & 2,74 & 0,72 & 3,20 & 0,65 & 1,61 & 0,28 & 1,80 & 0,30 & 2,11 & 0,51 \\
\hline $10^{\prime}$ & 3,45 & 0,71 & 4,00 & 0,80 & 1,90 & 0,29 & 2,20 & 0,40 & 2,41 & 0,30 \\
\hline $12^{\prime}$ & 4,32 & 0,87 & 4,62 & 0,62 & 2,13 & 0,23 & 2,20 & 0,40 & 2,95 & 0,53 \\
\hline $14^{\prime}$ & 5,10 & 0,78 & 5,20 & 0,58 & 2,22 & 0,19 & 2.84 & 0,24 & 3,18 & 0,23 \\
\hline $16^{\prime}$ & 5,70 & 0,60 & 5,60 & 0,40 & 2,45 & 0,23 & 3,21 & 0,37 & 3,40 & 0,32 \\
\hline $18^{\prime}$ & 6,40 & 0,70 & 5,90 & 0,30 & 2,67 & 0,22 & 3,57 & 0,36 & 3,71 & 9,31 \\
\hline $20^{\prime}$ & 7,02 & 0,62 & 6,20 & 0,30 & 2,87 & 0,20 & 3,81 & 0,24 & 4,02 & 0,31 \\
\hline
\end{tabular}


Wie aus Tabelle XIV hervorgeht, macht sich die Wirkung des Wechselstroms auf die Kontraktion erst dann deutlich bemerkbar, wenn das Verhältnis der Stromstärken von Gleichstrom zu Wechselstrom höchstens $1: 1$ ist, während eine Steigerung: der letzteren im Anfangsstadium der Abscheidung keine merkliche Verstärkung des Effektes, später vielleicht sogar eine Verringerung hervorzubringen scheint (Fig. 9).



Fig. 9.

Die sichtbare Struktur war beim Verhältnis $1: 0,5$ nicht verändert, mit steigender Wechselstromstärke wurde sie deutlich körniger und die Niederschläge lösten sich zunehmend schwerer in Säure auf.

Um festzustellen, wie sich dickere Niederschläge, die sonst die Erscheinung des Blätterns zeigen, unter dem Einfluss von Wechselstrom verhalten, wurden einige Dauerversuche, bei denen zwei gleiche Zellen mit und ohne Wechselstrom in denselben Gleichstromkreis geschaltet waren, angesetzt; die Elektrolyse blieb über Nacht in Gang, sodass sie im Durchschnitt 16 Stunden währte. In allen Fällen blieb das Blättern bei der Utberlagerung von Wechselstrom aus, während die Kathoden, die nur unter Gleichstrom gestanden hatten, stark verbogen und die abgelagerten Schichten stark aufgerissen waren. 
Tabelle XV.

\begin{tabular}{|c|c|c|c|}
\hline Nr. & $\begin{array}{c}\text { Ausgeschiedenes } \\
\text { Nickel }\end{array}$ & Verbältnis & Aussehen des Niederschlages \\
\hline \multicolumn{4}{|c|}{ n. $\mathrm{NiSO}_{4}, 0.5 \%$ Borsäure. Gleichstromdicbte $10 \mathrm{M.A.} / \mathrm{cm}^{2}$} \\
\hline \multirow[t]{2}{*}{130} & $0,6578 \mathrm{gr}$ & ohne W'strom & Stark verbogen und geblättert \\
\hline & 0,6397 & $1: 10$ & Glatt und nicht geblättert \\
\hline \multirow[t]{2}{*}{131} & 0,6509 , & ohne W'strom & rio \\
\hline & $0,6264 ”$ & $1: 5$ & wie open \\
\hline \multirow[t]{2}{*}{132} & 0,7096 & ohne W'strom & wie 130 \\
\hline & 0,7082 & $1: 2,5$ & \\
\hline \multirow{3}{*}{133} & b) n. $\mathrm{NiCl}_{2}$, & $\mathrm{NH}_{4} \mathrm{Cl} . \quad$ Gleichs & mdichte 10 M. A. $/ \mathrm{cm}^{2}$ \\
\hline & $0,6418 \mathrm{gr}$ & ohne W'strom & sehr stark geblättert \\
\hline & 0,4740 & $1: 10$ & vollständig glatt \\
\hline
\end{tabular}

Unter dem Mikroskop konnte man die vollständige Strukturänderung deutlich erkennen; die Ausscheidungen mit Wechselstrom waren alle grobkörnig, die anderen dagegen waren grau gefärbt und zeigten dichte Struktur.

Auch durch ihr chemisches Verhalten unterschieden sich beide deutlich voneinander, da die ersteren sich nur sehr langsam selbst in starken Säuren lösten und von 2-n. Ammoniaklösung bei Luftzutritt nicht angegriffen wurden, die letzteren aber durch Salpetersäure rasch, durch Salzsäure unter stürmischer Gasentwicklung und Ablösung in kleinen ,schwarzen Flocken in Lösung gingen, und von Ammoniak ebenfalls langsam gelöst wurden.

Zum Vergleich des mit Wechselstromüberlagerung abgeschiedenen Metalls mit anderen Nickelformen wurde schliesslich noch ein solcher Niederschlag neben einem Stück Walznickelblech und einer Probe reinen kompakten Nickels, anscheinend auch elektrolytischer Herkunft, bei annähernd gleichen Oberflächen unter 2-n. Salzsäure stehen gelassen; in 24 Stunden waren die beiden letztern unter Bildung tiefgrüner Lösungen merklich gelöst, das erstere aber nicht angegriffen, sodass die Frage aufgeworfen werden muss, ob nicht unter der Wirkung der positiven Wechselstromkomponente passiviertes Metall entsteht. Es wird hierauf in der nächsten Mitteilung zurückzukommen sein.

Die bei Zuschaltung von Wechselstrom erhaltene Nickelmenge ist, wie schon aus Tab. XV hervorgeht, immer geringer als 
in der Vergleichslösung. Über den Einfluss des Wechselstroms auf die Stromausbeute gibt die Tab. XVI noch genaueren Aufschluss; es zeigt sich, dass mit abnehmender Wechselstromdichte die Ausbeute sich verbessert und beim Verhältnis $1 \mathrm{zu} 1$ wenig von der normalen abweicht.

Tabelle XVI.

\begin{tabular}{|c|c|c|c|c|}
\hline $\mathrm{Nr}$ & $\begin{array}{c}\text { Verhältnis } \\
\text { Gl'strom : W'strom }\end{array}$ & $\begin{array}{c}\text { Ausgesehied. } \\
\text { Kupfer }\end{array}$ & \begin{tabular}{|} 
Ausgeschied. \\
Nickel
\end{tabular} & Ausbeute \\
\hline \multicolumn{5}{|c|}{ a) $\mathrm{n} . \mathrm{NiSO}_{4}+0,5 \%$ Borsäure. Gleichstromdichte $10 \mathrm{M}$. A. $/ \mathrm{cm}^{2}$} \\
\hline \multirow[t]{2}{*}{135} & ohne W'strom & $0,2956 \mathrm{gr}$ & $0,1700 \mathrm{gr}$ & $62,31 \%$ \\
\hline & $1: 10$ & & $0,1576 \eta$ & $57,77 \%$ \\
\hline \multirow[t]{2}{*}{136} & ohne W'strom & 0,3497 & 0,1751 & $58,93 \%$ \\
\hline & $1: 5$ & & 0,1428 & $54,25 \%$ \\
\hline \multirow[t]{2}{*}{137} & ohne W'strom & 0,2478 & $0,1330 "$ & $58,15 \%$ \\
\hline & $1: 2,5$ & & 0,1290 & $56,40 \%$ \\
\hline \multirow[t]{2}{*}{138} & ohne W'strom & $0,2921 \#$ & 0,1682 & $62,40 \%$ \\
\hline & $1: 1$ & & 0,1625 & $60,20 \%$ \\
\hline b) & \multicolumn{4}{|l|}{ n. $\mathrm{NiCl}_{2}+$ n. $\mathrm{NH}_{4} \mathrm{Cl}$} \\
\hline \multirow[t]{2}{*}{139} & ohne W'strom & $0,1357 \mathrm{gr}$ & $0.1159 \mathrm{gr}$ & $92,40 \%$ \\
\hline & $1: 10$ & & 0,0998 & $79,69 \%$ \\
\hline \multirow[t]{2}{*}{140} & ohne W'strom & $0,3222 "$ & 0,2737 , & $92,02 \%$ \\
\hline & $1: 5$ & & $0,2366 "$ & $79, \overline{5} 7 \% / 0$ \\
\hline \multirow[t]{2}{*}{141} & ohne W'strom & 0,2914 & 0,2485 & $92,42 \%$ \\
\hline & $1: 2,5$ & & $0,2351 \quad n$ & $85,50 \%$ \\
\hline \multirow[t]{2}{*}{142} & ohue W'strom & 0,2714 & 0,2318 & $92,41^{\circ} j 0$ \\
\hline & $1: 1$ & & $0,2206 n$ & $88,62 \%$ \\
\hline
\end{tabular}

Bei zusammenfassender Betrachtung der Versuche unter B und $\mathrm{C}$ ergibt sich, dass depolarisierende Wirkungen tatsächlich eine Veränderung der Kontraktionsfähigkeit und unmittelbar sichtbaren Struktur des elektrolytisch abgeschiedenen Nickels herbeiführen, wie es vorauszusetzen ist, wenn der gleichzeitig entladene Wasserstoff die letzte Ursache des Effektes ist. Der Gedanke, von dem die Untersuchung geleitet wurde, findet also auch hierdurch eine Bestätigung.

Bern, Anorganisches Laboratorium der Universität, April 1920. 